\title{
Open field mirror test as a tool for the assessment of visual functions in rats with streptozotocin-induced diabetes
}

Muhammad Zulfiqah Sadikan ${ }^{1}$, Nurul Alimah Abdul Nasir ${ }^{1 *}$, Igor lezhitsa ${ }^{2}$ and Renu Agarwal ${ }^{2}$

${ }^{1}$ Centre for Neuroscience Research (NeuRon), Faculty of Medicine, Universiti Teknologi MARA, Sungai Buloh, Selangor, 47000, Malaysia

${ }^{2}$ School of Medicine, International Medical University, Bukit Jalil, 57000 Kuala Lumpur, Malaysia

* Correspondence: nurulalimah@uitm.edu.my; Tel.: +60-03-6126-7230

Received: 26 May 2021; Accepted: 16 August 2021; Published: 28 August 2021

Edited by: Cheah Pike See (Universiti Putra Malaysia, Malaysia)

Reviewed by: Sangeeta Pilkhwal Sah (Panjab University, India); Norsyifa Binti Harun (Universiti Sains Malaysia, Malaysia); Wan Mastura Shaik Mohamed Mossadeq (Universiti Putra Malaysia, Malaysia)

https://doi.org/10.3117/neuroscirn.v4i3.74

\begin{abstract}
To evaluate the use of mirror test in an open field arena as a visual function assessment tool in a rodent model of diabetes. Male Sprague-Dawley rats were divided into diabetic rats, that received intraperitoneal streptozotocin ( $55 \mathrm{mg} / \mathrm{kg}$ body weight) for induction of diabetes, and control rats that similarly received citrate buffer. Rats with a blood glucose level of more than $20 \mathrm{mmol} / \mathrm{L}$ were considered diabetic. Blood glucose was monitored weekly throughout the experimental period. General behavioural assessment of the rats was done at week 12 post-induction using open field arena, followed by visualbehavioural assessment with mirror and reversed mirror added in the arena. Subsequently, rats were euthanised and subjected to haematoxylin and eosin staining (H\&E) staining to assess changes in retinal morphology. In the open field test, diabetic rats showed a lesser number of zone crossings (3.73-fold, $p<0.001)$, total distance travelled (2.02-fold, $p<0.001)$, number of rearing episodes $(2.22$-fold, $p<0.001)$ and number of grooming episodes $(4.33$-fold, $p<0.01)$ but a greater number of freezing episodes (2.47-fold, $p<0.001)$ and number of the faecal pellet $(4.17$-fold, $p<0.01)$ compared to control rats. Control rats spent more time with higher zone entries toward mirrored than non-mirrored and reversed mirror zones $(p<0.05$ and $p<0.01$ respectively), whereas diabetic rats showed no preference for zones. Normal rats also showed higher freezing episodes within the mirrored zone compared to diabetic rats $(2.00-f o l d, p<0.05)$. The retinal morphometry showed significant thinning of various retinal layers in the diabetic group compared to control rats. Visual behavioural activities of diabetic rats in an open field arena with the presence of a mirror could detect the presence of visual loss. Changes in visual functions positively correlated with changes in retinal morphology. Therefore, an open field mirror test could be used as an alternative for assessing visual function in the rodent model of diabetes.
\end{abstract}

Keywords: diabetes; visual function test; visual-behaviour response; open field test; mirror test;

(C)2021 by Sadikan et al. for use and distribution according to the Creative Commons Attribution (CC BY-NC 4.0) license (https://creativecommons.org/licenses/by-nc/4.0/), which permits unrestricted non-commercial use, distribution, and reproduction in any medium, provided the original author and source are credited.

\subsection{INTRODUCTION}

Diabetic retinopathy (DR) is one of the common microvascular complications of diabetes mellitus, which is expected to affect 160.50 million people in 2045 (Teo et al., 2021). Visual function deterioration with the progression of DR may lead to blindness. To understand the pathophysiology of DR and the development of future therapies to combat this disease, the use of an animal model is inevitable. The streptozotocin-induced rat model of diabetes is among the commonly used 
models to mimic DR and retinal neurodegeneration in humans (Abdul Nasir et al., 2017; Kohzaki et al., 2008; Tzeng et al., 2016).

Visual function tests have commonly been used as a tool for the assessment of retinal neurodegeneration in rodents. Direct visual-behavioural tests such as the optokinetic response test (Mdzomba et al., 2020) and trained visual-behaviour tests such as the two-direction water maze test (Prusky et al., 2000). The optokinetic response test objectively evaluates rodent visual acuity by tracking a stimulus moving from the temporal to nasal visual field (Douglas et al., 2005). The utility of this technique is controversial in albino rats because of functional deficits in their optokinetic system (Thomas et al., 2004).

The visual-behaviour test has rarely been used in rats with streptozotocin-induced DR due to difficulties in interpreting whether the likely cause of altered behavioural performance is attributable solely to visual changes. Firstly, behaviour test that requires strength and endurance such as water-maze is challenging for diabetic rodents as they are in catabolic condition, which may affect their performance (Etemad et al., 2015). Secondly, hyperglycaemia affects all organs including the brain and muscle which could alter their behaviour (Aparicio et al., 2016). These factors may contribute to poor performance which may inappropriately be attributed to poor visual functions. Therefore, in this study, we evaluated the use of the open field mirror test as a tool for the assessment of visual impairment in rats with streptozotocin-induced diabetes.

\subsection{MATERIALS AND METHODS}

\subsection{Animals and grouping}

All experiments were conducted at the Centre for Neurosciences (NeuRon), Faculty of Medicine, Universiti Teknologi MARA in accordance with the Association for Research in Vision and Ophthalmology (ARVO) statement for the use of animals in vision research. Institutional approval from UiTM Committee on Animal Research \& Ethics, UiTM CARE 3/2019/ (286/2019) was obtained. Sprague-Dawley male rats, weighing 200-250 grams ( 9 weeks old), were kept in the Laboratory Animal Care Unit (LACU) of Faculty of Medicine, Universiti Teknologi MARA on a 12-hour light/dark cycle with food and water available ad libitum. The rats were acclimatised for one week before starting the experiment and were examined for any systemic or ophthalmic abnormalities. Rats that showed no abnormalities were included in the experiment.

\subsection{Study design}

Animals were divided into two groups: healthy (control) rats $(n=8)$ and Streptozotocin-induced Diabetic Retinopathy (SIDR) rats $(n=10)$. The number of rats included in the SIDR group was higher due to the high mortality rate associated with this model. The rats in group 2 were subjected to induction of diabetes using streptozotocin. Behavioural tests, which included open field and mirror tests, were performed at twelve weeks post-diabetes induction. An open field test was done two days before euthanising the rats, whereas a mirror test was done on the day the rats were euthanised. Rats were then anaesthetised with an intraperitoneal injection of $0.14 \mathrm{mg} / \mathrm{kg}$ body weight Sodium Pentobarbital (Dolethal $^{\circledR}$, Vetoquinol UK Ltd, Northamptonshire, UK) and eyeballs were collected for assessment of retinal morphology using hematoxylin and eosin (H\&E) staining. This was followed by euthanisation through cardiac puncture.

\subsection{Induction of diabetes}

All animals were fasted overnight prior to induction of diabetes. Streptozotocin (Catalog No: sc-200719, Santa Cruz Biotechnology, Inc., Texas, US) solution was prepared in an ice-cold sodium citrate buffer (10 $\mathrm{mmol} / \mathrm{L}, \mathrm{pH}$ 4.5) and was given through a single intraperitoneal injection at a dose of $55 \mathrm{mg} / \mathrm{kg}$ body weight. Blood from the tail vein was collected 48 hours after injection and then weekly to measure blood glucose level using the Accu Chek Performa glucometer (Roche Diagnostic, Basel, $\mathrm{CH}$ ). The rats were considered diabetic if the blood glucose level was higher than 20 $\mathrm{mmol} / \mathrm{L}$ (Abdul Nasir et al., 2017; Cheng et al., 2006; Khathi et al., 2013) and were included in the study. The control group received a single intraperitoneal injection of $0.1 \mathrm{ml}$ of sodium citrate buffer.

\subsection{Assessment of exploratory behaviour in open field test}

The exploratory behaviour of rats was assessed in an open field test using a method described previously (Aizuddin Mohd Lazaldin et al., 2020; lezhitsa et al., 2011; Spasov et al., 2008). The observations were done in an open field test arena with dimensions $82 \times 82 \mathrm{~cm}$ enclosed by $25-\mathrm{cm}$ high white colour acrylic plastic walls. The whole arena was divided into peripheral and central zones (Figure 1A). All sessions were randomised with respect to control and diabetic rats a day before euthanasia. The rats were allowed to acclimate to the behavioural room environment for 24 hours and prehandled for 30 minutes before the testing commenced. The rats were evaluated once to avoid issues with 
habituation. All behavioural tests were performed between 0800 to 1200 to prevent any circadian related fluctuation with the performance of the animals. A digital video camera placed $2.5 \mathrm{~m}$ above the central zone was used to record experimental sessions. During the experiment, rats were placed at the centre of the arena. The following parameters were observed for 10 min:

(i) Total distance travelled

(ii) Frequency of freezing episodes (frequency at which the animal does not move for more than 2 seconds)

(iii) Frequency of rearing episodes (frequency at which the animal stood on its hind limbs)

(iv) Frequency of grooming episodes (frequency of licking and scratching)

(v) Number of faecal pellets excreted

(vi) Frequency of zone crossings

The open field arena was cleaned with $70 \%$ alcohol between test sessions to remove all sensory stimuli. The data was then analysed using Any Maze software version 4.0 (Stoelting Co., Wood Dale, Illinois, US).

\subsection{Assessment of visual impairment in the open field mirror test}

The design and procedure for the open field mirror test were adapted from the method earlier described by Naim et al. (2010). The open field arena was divided into three zones labelled $A, B$, and $C$, further divided into three subzones (Figure 1B). The dimension for each subzone was $27.33 \times 27.33 \mathrm{~cm}$. A mirror with dimensions of $25 \times 25 \mathrm{~cm}$ was placed in the middle of the wall in zone A. A reversed mirror of the same size with a non-reflective surface was placed on the opposite wall in Zone $C$, the surface of which mimicked the actual mirror except that it was not a reflective surface (Figure 1B). Zone B contained no mirror and was designated as a 'null zone'. All sessions were randomised with respect to control and diabetic rats on the day prior to euthanasia. For the open field test, the experimental sessions were recorded with a digital video camera. Each rat was placed in the central part of the open field arena. The following behavioural parameters were observed for 10 minutes:

(i) Total distance travelled

(ii) Time spent in the mirror-, reversed mirror- and null zones

(iii) Frequency of zone entries

(iv) Frequency of freezing episodes
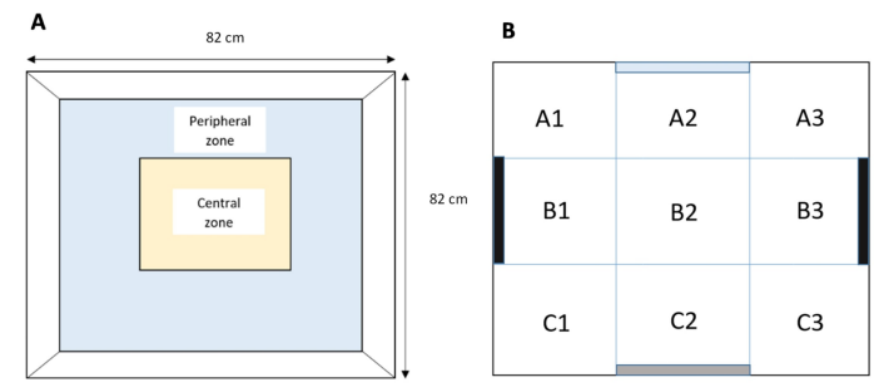

Figure 1: Representative diagrams showing the set-up of equipment for open field mirror test. (A) The open field test arena with two zones: central and peripheral; (B) Mirror placement in the open field arena. A2: mirrored area, C2: reversed mirror, $\mathrm{B} 1$ and $\mathrm{B} 3$ : null zones.

\subsection{Assessment of retinal morphology}

Eyeballs were enucleated and fixed in $10 \%$ neutral buffered formalin for 24 hours, followed by paraffin embedding. Eyeballs were sectioned at $1 \mathrm{~mm}$ from the temporal edge of the optic disc with $3 \mu \mathrm{M}$ thickness. The tissue sections were then stained with H\&E. The stained retinal sections were examined by two independent observers using a light microscope (Olympus IX8, Olympus Corporation, Tokyo, Japan). The retinal sections $(n=8)$ were obtained at $1 \mathrm{~mm}$ from the temporal edge of the optic disk. Five randomly selected areas of $3 \times 10^{6} \mu \mathrm{m}^{2}$ from each section were captured using imaging software (NIS-Elements Basic Research, version 4.30, Nikon Instrument Inc., Tokyo, Japan). The thickness of the inner retina (IR), outer retina (OR), ganglion cell layer $(\mathrm{GCL})$, and total retina were measured using Image J software (Image J 1.31, National Institutes of Health, Bethesda, MD, USA).

\subsection{Statistical analysis}

Data from both open field test and morphometric analysis was presented as mean \pm standard deviation (SD) and was analysed using an unpaired T-test (SPSS 22.0, SPSS Inc., Chicago, IL). In contrast, data from the mirror test was presented as mean \pm standard error of the mean (SEM) and was analysed using the KruskalWallis $H$ test. A p-value less than 0.05 was considered statistically significant.

\subsection{RESULTS}

\subsection{Blood glucose level}

During the experiment, two rats from the diabetic group were found dead. Hence, there were 8 rats in the normal group and 8 rats in the SIDR group $(n=8)$. The blood glucose level in diabetic rats remained significantly higher compared to control rats starting from 48 hours post-STZ injection until the end of the 


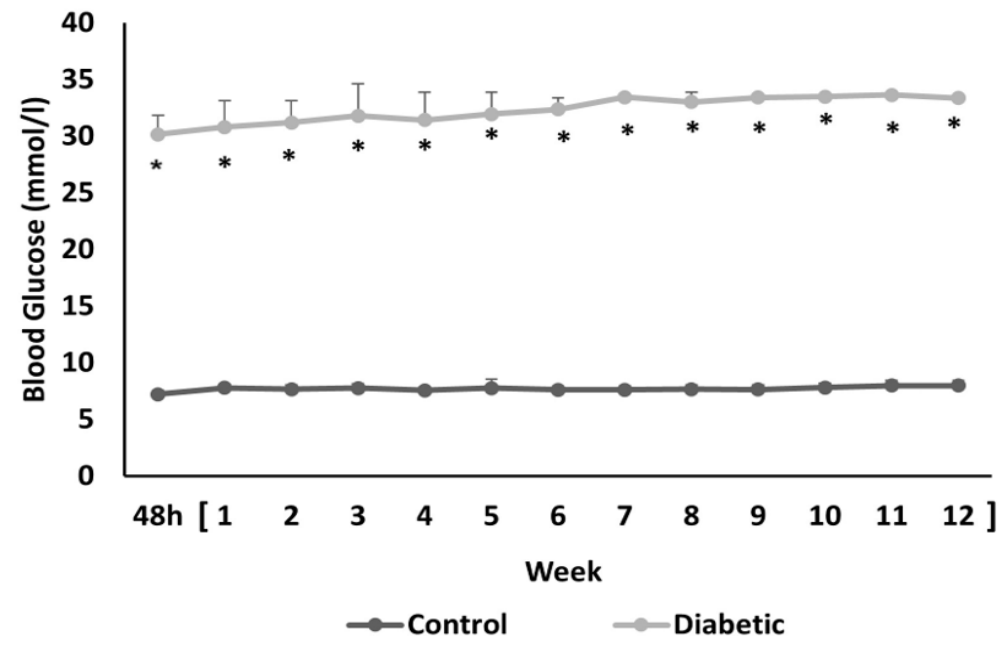

Figure 2: The blood glucose level of diabetic rats compared to control rats during 12 weeks of experimental period. $\mathrm{n}=8,{ }^{*} p<0.001$ compared to control group
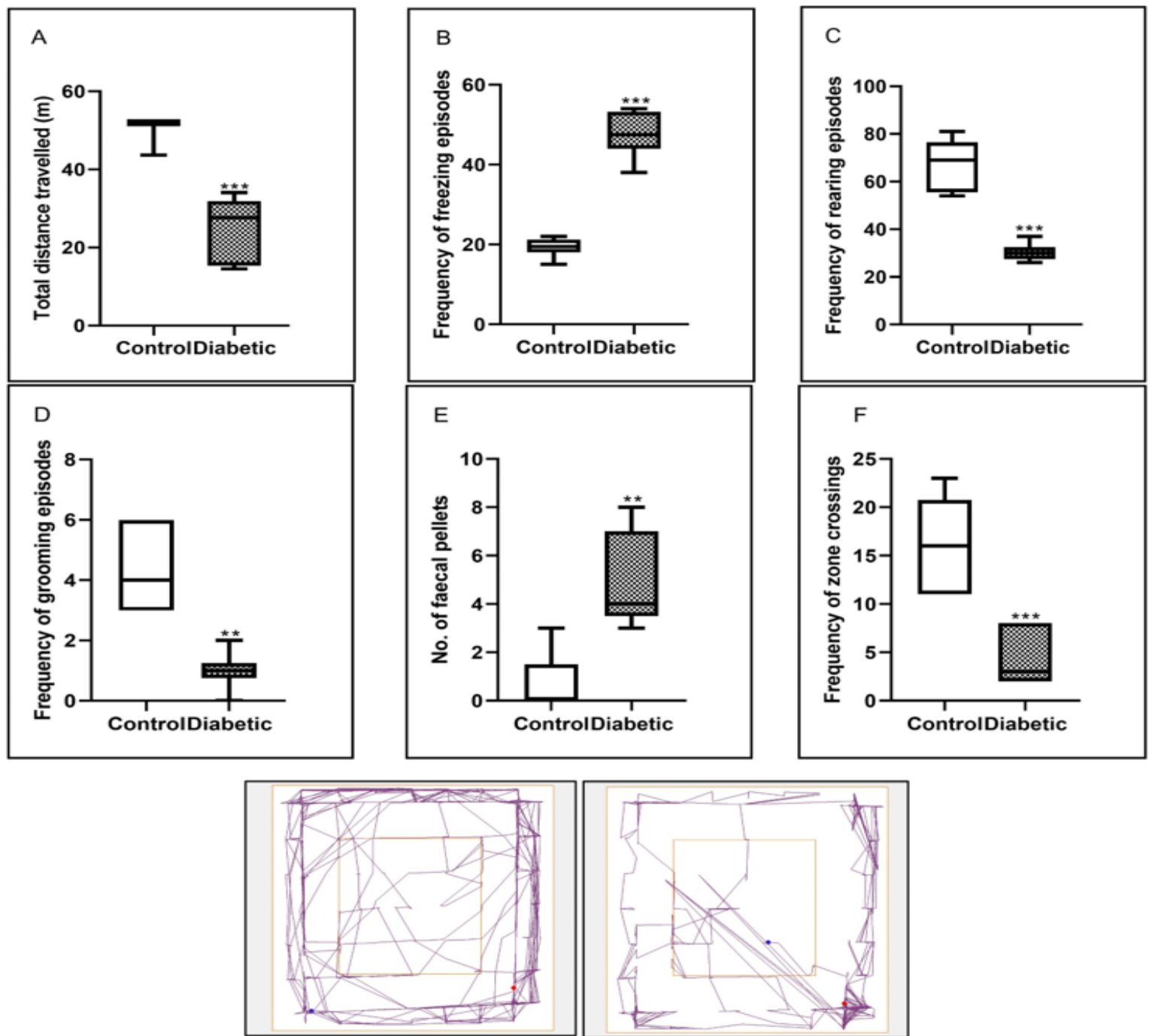

Control

Diabetic

Figure 3: The task performance by rats with streptozotocin-induced diabetes compared to control rats in open field test: (A) Total distance travelled; (B) Frequency of freezing episodes; (C) Frequency of rearing episodes; (D) Frequency of grooming episodes; (E) Number of faecal pellet; and (F) Frequency of zone crossings. Differences in the travelling pattern of rats is shown in the track plot for both control and diabetic groups. $\mathrm{n}=8,{ }^{* *} p<0.01, * * * p<0.001$. 
experimental period $(p<0.001)$. The mean blood glucose for control rats at week 12 of the experiment was $8.0 \pm 0.47 \mathrm{mmol} / \mathrm{L}$ compared to $33.3 \pm 0.18 \mathrm{mmol} / \mathrm{L}$ in the SIDR group (Figure 2).

\subsection{Open field test}

The STZ-induced diabetic rats demonstrated the lesser frequency of zone crossings ( 3.73 -fold, $p<0.001$ ), total distance travelled $(2.02$-fold, $p<0.001)$, frequency of rearing episodes $(2.22$-fold, $p<0.001)$ and frequency of grooming episodes $(4.33-$ fold, $p<0.01)$ compared to rats in the control group. Additionally, diabetic rats showed a greater frequency of freezing episodes (2.47-fold, $p<0.001)$ and the number of the faecal pellet $(4.17$-fold, $p<0.01$ ) compared to the control rats (Figure 3 ). Comparison of the track plots for control rats revealed uninhibited exploratory activity. In contrast, the diabetic rats showed relatively lesser exploratory activity that was especially notable in the peripheral zone of the open field arena.

\subsection{Mirror test}

In this study diabetic rats did not demonstrate any zone preference whereas control rats showed a higher preference for zone A2. The controls rats had greater frequency of entries to zone $A 2$, total time spent, distance travelled and freezing episodes in zone A2 compared to diabetic rats (Figure 4).

\subsection{Retinal morphology}

The thickness of the various retinal layer was measured using H\&E-stained retinal sections (Figure 5A). The thickness of IR, OR, GCL and total retina was lesser in SIDR by 1.39-, 1.54-, 1.86- and 1.45-fold respectively, compared to the normal control rats $(p<0.05)$ (Figure 5B).

\subsection{DISCUSSION}

Retinal dysfunction following prolonged periods of hyperglycaemia leads to visual disturbances such as loss of visual field, visual acuity, colour sensitivity and contrast sensitivity (Bao et al., 2019; Henricsson \& Heijl, 1994; Maguire et al., 2020). In the experimental setting, it is important to have visual function assessment tests that could help to evaluate changes in visual functions of rats with diabetes and associated retinopathy.

In this study, we assessed the utility of the Open Field Mirror Test described earlier by Naim et al. (2010) using piglets as a tool for the assessment of visual impairment in rats with streptozotocin-induced diabetes. Open field test is commonly used in behavioural studies and is accepted as a standard tool to assess rodents' exploratory behaviour and general activity since its introduction for the first time by Hall \& Ballachey (1932). In this study, we observed slower locomotor and exploratory activities in diabetic rats, which included lesser zone crossings, distance travelled, and total rearing compared to control rats. The anxiety-related behavioural patterns were also enhanced in diabetic rats as evidenced by a lower number of grooming episodes, a higher number of freezing episodes and faecal pellets excreted. Similar observations were seen in several behavioural studies conducted earlier using rat models of STZ-induced diabetes (Ergenc et al., 2019; Rajashree et al., 2011; Tang et al., 2015). Several factors were suggested to be involved in anxiety-related behaviour in diabetic rats. Among them most notable are reduction of serotonin synthesis and metabolism (Thorré et al., 1997), dysregulation of neurotrophic factors influenced by C-peptide deficiency (Sima et al., 2009) and chronic inflammatory responses towards neurons and synapses due to insulin deficiency (Rajashree et al., 2011). However, the actual pathophysiological mechanism of diabetes-induced anxiety remains unclear (Rajabi et al., 2018).

In Open Field Mirror Test, control rats spent more time in front of the mirror compared to null zones and zone with reversed mirrors. This may be the reason they exhibited more freezing episodes while in front of the mirror. Naim et al. (2010) and Broom et al. (2009) observed a similar type of behaviour in normal piglets, which suggests that animals with intact visual function could recognise the reflection in the mirror. Rats have been found to have good visual recognition $\underline{\underline{H} \text { ölscher et }}$ al., 2005). Rats also can distinguish between conspecific rat and their reflection in the mirror and are known to be aware of the changes in their surroundings (Hills \& Butterfill, 2015). However, it is unclear whether rats perceive mirror images of themselves as their images (Ueno et al., 2020). As was observed by Naim et al. (2010) and Broom et al. (2009) for piglets, we also noted that control rats showed considerably greater interest in interacting with the mirror than with the other novel object, the reversed mirror.

Interestingly, control rats also showed higher exploratory activity in $\mathrm{A} 1$ and $\mathrm{A} 3$ zones, situated next to the mirror zone. This could be explained by the fact that rats have a wide visual field (Wallace et al., 2013) despite having low visual acuity (Prusky et al., 2000). In fact, rats have a large panoramic field of view extending in front, above and behind the animal's head (Wallace et al., 2013) and that perhaps made them see the reflection in the mirror even from $\mathrm{A} 1$ and $\mathrm{A} 3$ zones. 
No zone preferences were observed among diabetic rats and time spent in all zones was almost equal. Observed behaviour was not associated with habituation to an increasingly familiar environment as the rats were not subjected to prior training and a single session of test was performed over 10 minutes.
Therefore, it seems that the diabetic rats have some degree of visual loss that affects their ability to recognise themselves via the mirror. This is likely to result in their inability to distinguish one novel object, mirror, from the other novel object, reversed mirror.
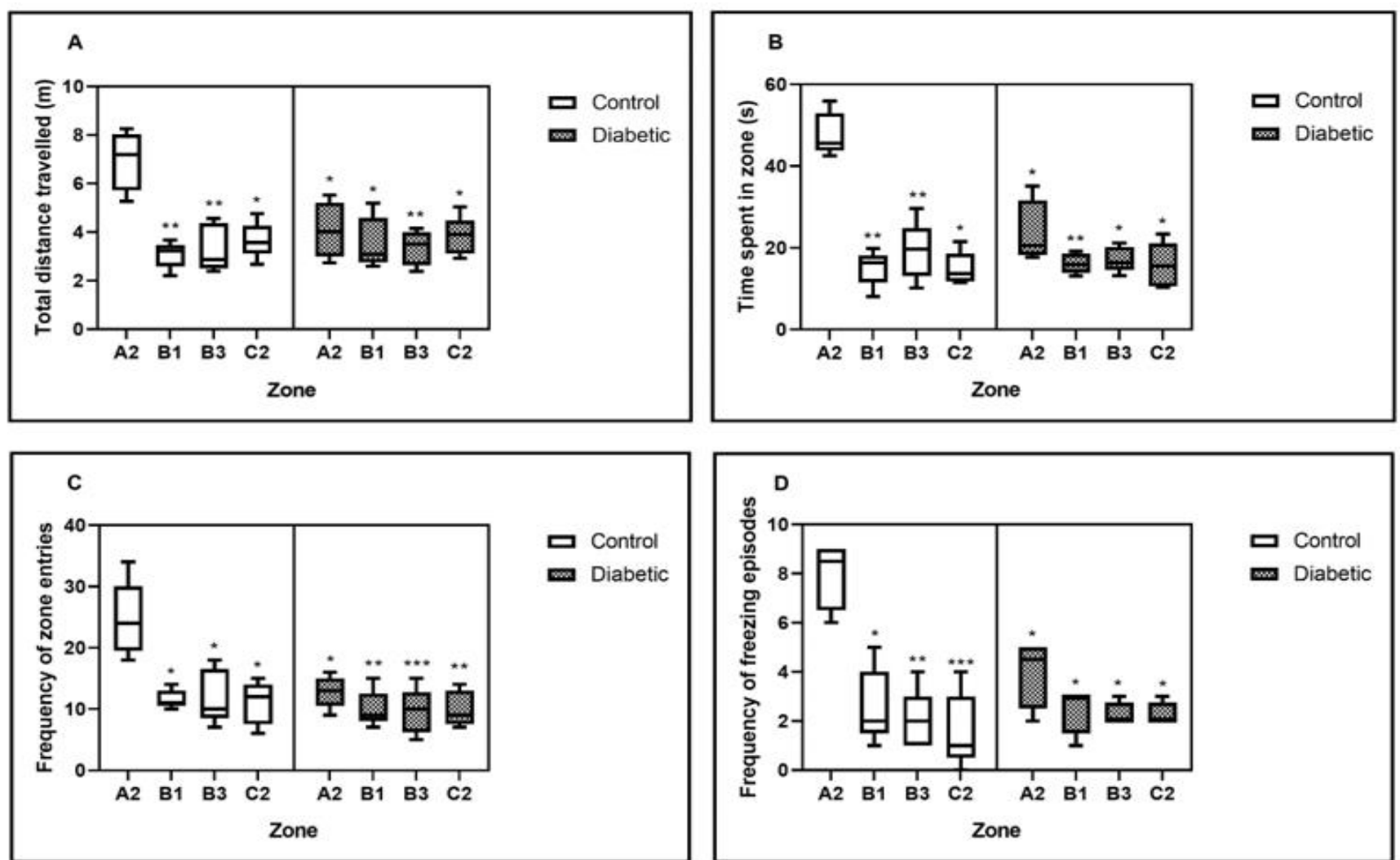

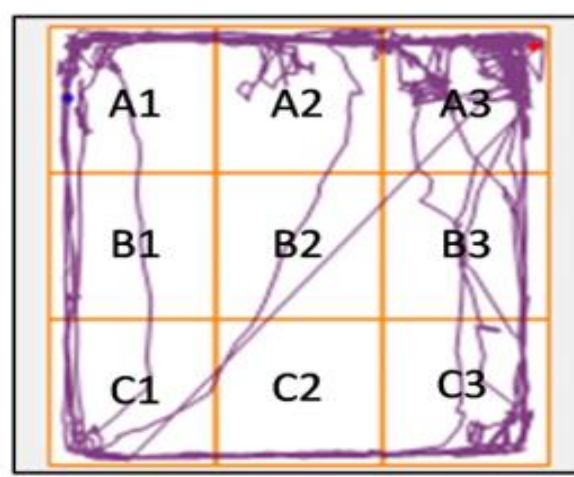

Control

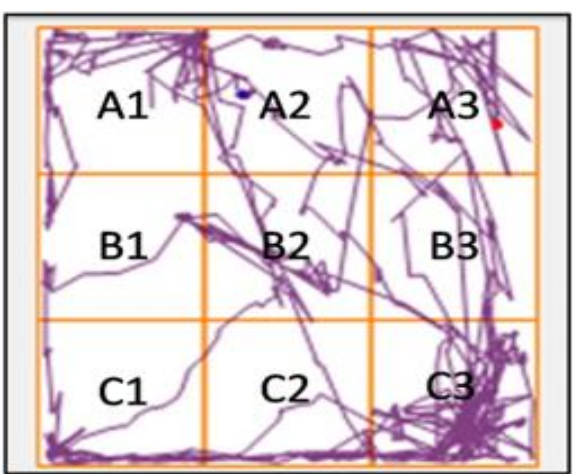

Diabetic

Figure 4: The task performance by rats with streptozotocin-induced diabetes compared to control rats in mirror test: (A) Total distance travelled; (B) Time spent in various zone (C) Frequency of zone entries; and (D) Frequency of freezing episodes. Differences in the travelling pattern of rats is shown in the track plot for both control and diabetic groups. $n=8, * p<0.05, * *$ $p<0.01, * * * p<0.001$ 
The abnormal visual behaviour activities seen in diabetic rats in this study corresponded with the retinal morphometric changes. The retinal morphometric analysis showed that various retinal layers were significantly thinner compared to normal control rats. Similar observation on the retinal layer thickness was made in several other studies using a rodent model of STZ-induced DR (Kim et al., 2016; Ozaki et al., 2018; Yang et al., 2015). Structural neurodegenerative alterations such as neural apoptosis, ganglion cells death, and reduction in retinal layer thickness have been found in the earliest stages of DR (Tang et al., 2020). DR manifests clinically with microvascular changes; however, studies have shown that disruption of the neurovascular unit occurs early in the disease process and leads to neurodegeneration, which includes reduced neuronal functions and neural cell apoptosis. The retinal ganglion cells, amacrine cells, and also photoreceptors, undergo apoptosis which reflects morphological changes in the retina including reduction of the thickness of its various layers (Tonade \& Kern, 2020). Hence, thinning of retinal layers is an important morphological change, indicating neurodegeneration and this has been confirmed by optical coherence tomography (OCT) in the early phase of DR (Jiang et al., 2018). In the current study, we observed significant thinning of various layers of the retina in diabetic rats
A

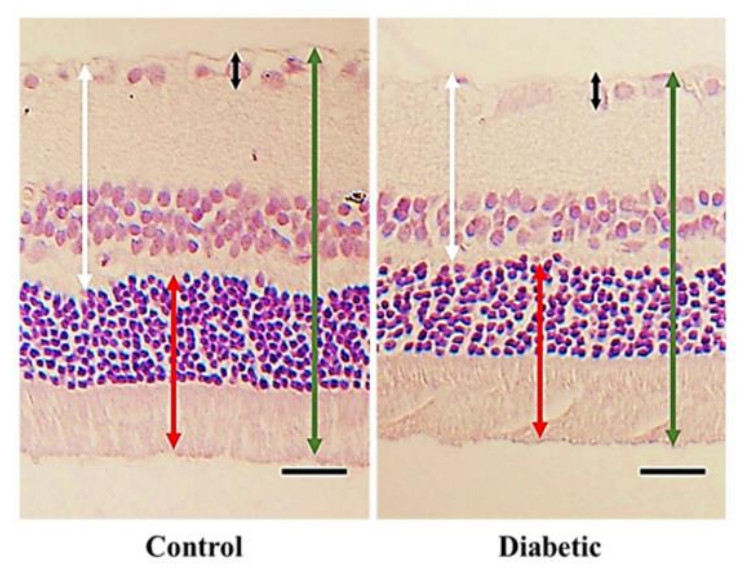

B

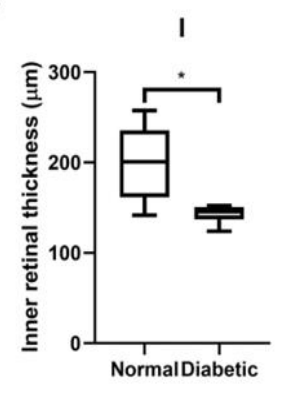

॥

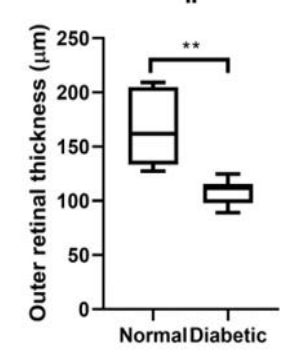

III

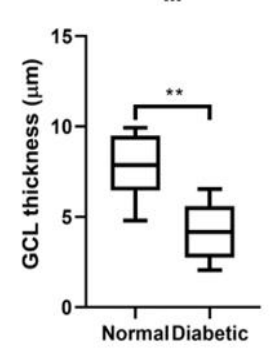

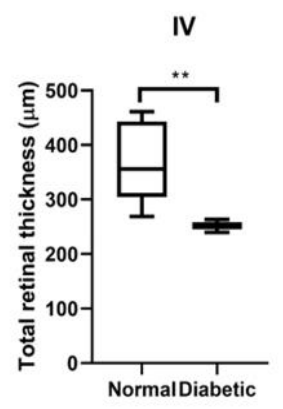

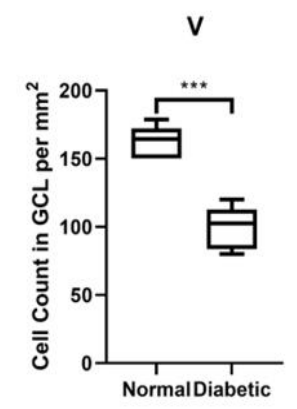

Figure 5: (A) Microphotograph of H\&E-stained retinal sections showing the thickness of various retinal layers in diabetic rats
compared to control rats (magnification $20 \times$ ). White arrow: thickness of inner retina (IR); Red arrow: Thickness of outer retina
(OR); Black arrow: Thickness of ganglion cell layer (GCL); Green arrow: total retinal thickness. $\mathrm{n}=8$ (Scale bar: $50 \mu \mathrm{m}$ ). (B) The
thickness of various retinal layers of diabetic rats compared to control rats (I) IR, (II) OR, (III) GCL, (IV) Total retina and (V)
Number of cell count in GCL are presented using box and whisker plots. Boxes show median, lower quartile, and upper quartile,
whereas whiskers show the data variability outside the upper and lower quartiles. $\mathrm{n}=8, * p<0.05, * * p<0.01, * * * p<0.001$.

compared to control rats presumably due to loss of neural elements. Other studies have also shown that the induction of diabetes in rats is associated with reduced thickness of various retinal layers (Ali et al., 2019; He et al., 2019; Zhang et al., 2020). The structural changes translate into functional alterations and have clinical consequences in terms of defects in a sensory capacity, involving reduced hue discrimination, colour sensitivity, delayed dark adaptation, and abnormal visual fields. In the current study, consequences of retinal morphological changes seem to translate into altered visual-behaviour responses (Simó et al., 2018; Trento et al., 2017).

Visual loss in diabetic rodents has been observed as early as one month after STZ injection (Aung et al., 2014). Retinopathy in a streptozotocin-induced model of diabetes is associated with the damage of retinal vasculature which could result in haemorrhages, macular oedema, and neovascularisation (Jackson \& 
Barber, 2010). As such, evaluation of visual function is an important step for the assessment of STZ-induced DR.

\subsection{CONCLUSIONS}

Advantages of open field mirror test is the simplicity of design and procedure, which does not require specialised techniques and enables the rapid assessment of visual function in rats. Additionally, this method does not require animal training prior to the main experiment, making it useful not only for assessing visual impairment but also for screening potentially retinoprotective drugs. Notably, the poor muscle strength of animals is not a significant limitation in mirror test compared to water maze test. In the water maze test, impaired muscle strength may contribute to poor task performance by animals which may be misinterpreted as the deficiency of visual functions. Considering these benefits, the mirror test described in this study appears to have an advantage to be used as an alternative or additional visual function assessment tools, particularly for diabetic rats.
Acknowledgements: This work was supported by Universiti Teknologi MARA (UiTM) grants (600-IRMI/DANA 5/3/BESTARI (P) (001/2018) and 600-IRMI/DANA 5/3/BESTARI (P) (012/2018)) and Ministry of Education (MOE), MY grant (600IRMI/FRGS 5/3 (101/2019)). Authors acknowledge the Center for Neuroscience Research (NeuRon), Institute of Medical Molecular Biotechnology (IMMB), and Laboratory Animal Care Unit (LACU), Faculty of Medicine, UiTM Sg Buloh for facility support.

Author Contributions: All authors of this research paper have directly participated in the formal analysis and methodology, N.A.A.N., I.I., R.A. were contributed to conceptualisation, supervision, validation and writing - review \& editing, N.A.A.N. and R.A. involved in funding acquisition, N.A.A.N. and M.Z.S have participated in project administration, N.A.A.N. was involved in finding resources and involved in visualisation and M.Z.S was carried out investigation, data curation and writing - original draft.

Conflicts of Interest: The authors declare no conflict of interest.

\section{References}

Abdul Nasir, N. A., Agarwal, R., Sheikh Abdul Kadir, S. H., Vasudevan, S., Tripathy, M., lezhitsa, I., Mohammad Daher, A., Ibrahim, M. I., \& Mohd Ismail, N. (2017). Reduction of oxidative-nitrosative stress underlies anticataract effect of topically applied tocotrienol in streptozotocin-induced diabetic rats. PloS One, 12(3), e0174542. https://doi.org/10.1371/journal.pone.0174542

Aizuddin Mohd Lazaldin, M., lezhitsa, I., Agarwal, R., Salmah Bakar, N., Agarwal, P., \& Mohd Ismail, N. (2020). Neuroprotective effects of brain-derived neurotrophic factor against amyloid beta 1-40-induced retinal and optic nerve damage. European Journal of Neuroscience, 51(12), 2394-2411. https://doi.org/10.1111/ejn.14662

Ali, S. A., Zaitone, S. A., Dessouki, A. A., \& Ali, A. A. (2019). Pregabalin affords retinal neuroprotection in diabetic rats: Suppression of retinal glutamate, microglia cell expression and apoptotic cell death. Experimental Eye Research, 184, 7890. https://doi.org/10.1016/i.exer.2019.04.014

Aparicio, V., Coll-Risco, I., Camiletti-Moirón, D., Nebot, E., Martínez, R., López-Jurado, M., \& Aranda, P. (2016). Interval aerobic training combined with strength-endurance exercise improves metabolic markers beyond caloric restriction in Zucker rats. Nutrition, Metabolism and Cardiovascular Diseases, 26(8), 713-721. https://doi.org/10.1016/j.numecd.2016.01.005

Aung, M. H., na Park, H., Han, M. K., Obertone, T. S., Abey, J., Aseem, F., Thule, P. M., Iuvone, P. M., \& Pardue, M. T. (2014). Dopamine deficiency contributes to early visual dysfunction in a rodent model of type 1 diabetes. Journal of Neuroscience, 34(3), 726-736. https://doi.org/10.1523/JNEUROSCI.3483-13.2014

Bao, Y. K., Yan, Y., Gordon, M., McGill, J. B., Kass, M., \& Rajagopal, R. (2019). Visual Field Loss in Patients With Diabetes in the Absence of Clinically-Detectable Vascular Retinopathy in a Nationally Representative Survey. Investigative Ophthalmology \& Visual Science, 60(14), 4711-4716. https://doi.org/10.1167/iovs.19-28063

Broom, D. M., Sena, H., \& Moynihan, K. L. (2009). Pigs learn what a mirror image represents and use it to obtain information. Animal Behaviour, 78(5), 1037-1041. https://doi.org/10.1016/i.anbehav.2009.07.027

Cheng, J. T., Huang, C. C., Liu, I. M., Tzeng, T. F., \& Chang, C. J. (2006). Novel mechanism for plasma glucose-lowering action of metformin in streptozotocin-induced diabetic rats. Diabetes, 55(3), 819-825. https://doi.org/10.2337/diabetes.55.03.06.db05-0934

Douglas, R., Alam, N., Silver, B., McGill, T., Tschetter, W., \& Prusky, G. (2005). Independent visual threshold measurements in the two eyes of freely moving rats and mice using a virtual-reality optokinetic system. Visual Neuroscience, 22(5), 677684. https://doi.org/10.1017/S0952523805225166

Ergenc, M., Ozacmak, H. S., Turan, I., \& Ozacmak, V. H. (2019). Melatonin reverses depressive and anxiety like-behaviours induced by diabetes: involvement of oxidative stress, age, rage and S100B levels in the hippocampus and prefrontal cortex of rats. Archives of Physiology and Biochemistry, 1-9. https://doi.org/10.1080/13813455.2019.1684954 
Etemad, A., Sheikhzadeh, F., \& Asl, N. A. (2015). Evaluation of brain-derived neurotrophic factor in diabetic rats. Neurological Research, 37(3), 217-222. https://doi.org/10.1179/1743132814Y.0000000428

Hall, C., \& Ballachey, E. L. (1932). A study of the rat's behavior in a field. A contribution to method in comparative psychology. University of California Publications in Psychology.

He, M., Long, P., Guo, L., Zhang, M., Wang, S., \& He, H. (2019). Fushiming capsule attenuates diabetic rat retina damage via antioxidation and anti-inflammation. Evidence-Based Complementary and Alternative Medicine, 2019. https://doi.org/10.1155/2019/5376439

Henricsson, M., \& Heijl, A. (1994). Visual fields at different stages of diabetic retinopathy. Acta Ophthalmologica, 72(5), 560569. https://doi.org/10.1111/i.1755-3768.1994.tb07180.x

Hills, T. T., \& Butterfill, S. (2015). From foraging to autonoetic consciousness: The primal self as a consequence of embodied prospective foraging. Current Zoology, 61(2), 368-381. https://doi.org/10.1093/czoolo/61.2.368

Hölscher, C., Schnee, A., Dahmen, H., Setia, L., \& Mallot, H. A. (2005). Rats are able to navigate in virtual environments. Journal of Experimental Biology, 208(3), 561-569. https://doi.org/10.1242/jeb.01371

lezhitsa, I. N., Spasov, A. A., Kharitonova, M. V., \& Kravchenko, M. S. (2011). Effect of magnesium chloride on psychomotor activity, emotional status, and acute behavioural responses to clonidine, d-amphetamine, arecoline, nicotine, apomorphine, and L-5-hydroxytryptophan. Nutritional Neuroscience, 14(1), 10-24. https://doi.org/10.1179/174313211X12966635733277

Jackson, G. R., \& Barber, A. J. (2010). Visual dysfunction associated with diabetic retinopathy. Current Diabetes Reports, 10(5), 380-384. https://doi.org/10.1007/s11892-010-0132-4

Jiang, J., Liu, Y., Chen, Y., Ma, B., Qian, Y., Zhang, Z., Zhu, D., Wang, Z., \& Xu, X. (2018). Analysis of changes in retinal thickness in type 2 diabetes without diabetic retinopathy. Journal of Diabetes Research, 2018. https://doi.org/10.1155/2018/3082893

Khathi, A., Serumula, M. R., Myburg, R. B., Van Heerden, F. R., \& Musabayane, C. T. (2013). Effects of Syzygium aromaticumderived triterpenes on postprandial blood glucose in streptozotocin-induced diabetic rats following carbohydrate challenge. PLoS One, 8(11), e81632. https://doi.org/10.1371/journal.pone.0081632

Kohzaki, K., Vingrys, A. J., \& Bui, B. V. (2008). Early inner retinal dysfunction in streptozotocin-induced diabetic rats. Investigative Ophthalmology \& Visual Science, 49(8), 3595-3604. https://doi.org/10.1167/iovs.08-1679

Kim, S.-J., Yoo, W.-S., Choi, M., Chung, I., Yoo, J.-M., \& Choi, W.-S. (2016). Increased O-GlcNAcylation of NF-kB enhances retinal ganglion cell death in streptozotocin-induced diabetic retinopathy. Current Eye Research, 41(2), 249-257. https://doi.org/10.3109/02713683.2015.1006372

Maguire, M. G., Liu, D., Glassman, A. R., Jampol, L. M., Johnson, C. A., Baker, C. W., Bressler, N. M., Gardner, T. W., Pieramici, D., \& Stockdale, C. R. (2020). Visual Field Changes Over 5 Years in Patients Treated With Panretinal Photocoagulation or Ranibizumab for Proliferative Diabetic Retinopathy. JAMA Ophthalmology, 138(3), 285-293. https://doi.org/10.1001/jamaophthalmol.2019.5939

Mdzomba, J. B., Joly, S., Rodriguez, L., Dirani, A., Lassiaz, P., Behar-Cohen, F., \& Pernet, V. (2020). Nogo-A-targeting antibody promotes visual recovery and inhibits neuroinflammation after retinal injury. Cell Death \& Disease, 11(2), 1-16. https://doi.org/10.1038/s41419-020-2302-x

Naim, M. Y., Friess, S., Smith, C., Ralston, J., Ryall, K., Helfaer, M. A., \& Margulies, S. S. (2010). Folic acid enhances early functional recovery in a piglet model of pediatric head injury. Developmental Neuroscience, 32(5-6), 466-479. https://doi.org/10.1159/000322448

Ozaki, H., Inoue, R., Matsushima, T., Sasahara, M., Hayashi, A., \& Mori, H. (2018). Serine racemase deletion attenuates neurodegeneration and microvascular damage in diabetic retinopathy. PloS One, 13(1), 1-11. https://doi.org/10.1371/journal.pone.0190864

Prusky, G. T., West, P. W., \& Douglas, R. M. (2000). Behavioral assessment of visual acuity in mice and rats. Vision Research, 40(16), 2201-2209. https://doi.org/10.1016/s0042-6989(00)00081-x

Rajabi, M., Mohaddes, G., Farajdokht, F., Nayebi Rad, S., Mesgari, M., \& Babri, S. (2018). Impact of loganin on proinflammatory cytokines and depression-and anxiety-like behaviors in male diabetic rats. Physiology International, 105(2), 116-126. https://doi.org/10.1556/2060.105.2018.1.8

Rajashree, R., Kholkute, S. D., \& Goudar, S. S. (2011). Effects of duration of diabetes on behavioural and cognitive parameters in streptozotocin-induced juvenile diabetic rats. The Malaysian Journal of Medical Sciences, 18(4), 26-31.

Sima, A. A., Zhang, W., Muzik, O., Kreipke, C. W., Rafols, J. A., \& Hoffman, W. H. (2009). Sequential abnormalities in type 1 diabetic encephalopathy and the effects of C-peptide. The Review of Diabetic Studies, 6(3), 211-222. https://doi.org/10.1900/RDS.2009.6.211

Simó, R., Stitt, A. W., \& Gardner, T. W. (2018). Neurodegeneration in diabetic retinopathy: does it really matter? Diabetologia, 61(9), 1902-1912. https://doi.org/10.1007/s00125-018-4692-1

Spasov, A., lezhitsa, I., Kharitonova, M., \& Kravchenko, M. (2008). Depression-like and anxiety-related behaviour of rats fed with magnesium-deficient diet. Zhurnal vysshei nervnoi deiatelnosti imeni IP Pavlova, 58(4), 476-485. 
Tang, Z.-J., Zou, W., Yuan, J., Zhang, P., Tian, Y., Xiao, Z.-F., Li, M.-H., Wei, H.-J., \& Tang, X.-Q. (2015). Antidepressant-like and anxiolytic-like effects of hydrogen sulfide in streptozotocin-induced diabetic rats through inhibition of hippocampal oxidative stress. Behavioural Pharmacology, 26(5), 427-435. https://doi.org/10.1097/FBP.0000000000000143

Tang, Z., Chan, M. Y., Leung, W. Y., Wong, H. Y., Ng, C. M., Chan, V. T., Wong, R., Lok, J., Szeto, S., \& Chan, J. C. (2020). Assessment of retinal neurodegeneration with spectral-domain optical coherence tomography: a systematic review and meta-analysis. Eye, 1-9. https://doi.org/10.1038/s41433-020-1020-z

Teo, Z. L., Tham, Y. C., Yu, M. C. Y., Chee, M. L., Rim, T. H., Cheung, N., ... \& Cheng, C. Y. (2021). Global Prevalence of Diabetic Retinopathy and Projection of Burden through 2045: Systematic Review and Meta-analysis. Ophthalmology, 2021, https://doi.org/10.1016/i.ophtha.2021.04.027.

Thomas, B. B., Seiler, M. J., Sadda, S. R., Coffey, P. J., \& Aramant, R. B. (2004). Optokinetic test to evaluate visual acuity of each eye independently. Journal of Neuroscience Methods, 138(1-2), 7-13. https://doi.org/10.1016/i.jneumeth.2004.03.007

Thorré, K., Chaouloff, F., Sarre, S., Meeusen, R., Ebinger, G., \& Michotte, Y. (1997). Differential effects of restraint stress on hippocampal 5-HT metabolism and extracellular levels of 5-HT in streptozotocin-diabetic rats. Brain Research, 772(1-2), 209-216. https://doi.org/10.1016/s0006-8993(97)00841-x

Tonade, D., \& Kern, T. S. (2020). Photoreceptor cells and RPE contribute to the development of diabetic retinopathy. Progress in Retinal and Eye Research, 83, 100919. https://doi.org/10.1016/j.preteyeres.2020.100919

Trento, M., Durando, O., Lavecchia, S., Charrier, L., Cavallo, F., Costa, M. A., Hernández, C., Simó, R., Porta, M., \& Investigators, E. T. (2017). Vision related quality of life in patients with type 2 diabetes in the EUROCONDOR trial. Endocrine, 57(1), 83-88. https://doi.org/10.1007/s12020-016-1097-0

Tzeng, T. F., Liu, W. Y., Liou, S. S., Hong, T. Y., \& Liu, I. M. (2016). Antioxidant-rich extract from plantaginis semen ameliorates diabetic retinal injury in a streptozotocin-induced diabetic rat model. Nutrients, 8(9), 572. https://doi.org/10.3390/nu8090572

Ueno, H., Suemitsu, S., Murakami, S., Kitamura, N., Wani, K., Takahashi, Y., ... \& Ishihara, T. (2020). Behavioural changes in mice after getting accustomed to the mirror. Behavioural Neurology, 2020, 4071315. https://doi.org/10.1155/2020/4071315

Wallace, D. J., Greenberg, D. S., Sawinski, J., Rulla, S., Notaro, G., \& Kerr, J. N. (2013). Rats maintain an overhead binocular field at the expense of constant fusion. Nature, 498(7452), 65-69. https://doi.org/10.1038/nature12153

Yang, Q., Xu, Y., Xie, P., Cheng, H., Song, Q., Su, T., Yuan, S., \& Liu, Q. (2015). Retinal neurodegeneration in db/db mice at the early period of diabetes. Journal of Ophthalmology, 2015(1), 1-9. https://doi.org/10.1155/2015/757412

Zhang, X., Peng, L., Dai, Y., Sheng, X., Chen, S., \& Xie, Q. (2020). Effects of Coconut Water on Retina in Diabetic Rats. EvidenceBased Complementary and Alternative Medicine, 2020, 9450634. https://doi.org/10.1155/2020/9450634 